\title{
Avaliação de Competências em um Sistema de Recomendação de Objetos de Aprendizagem
}

\author{
Ketia Kellen Araújo da Silva, UFRGS, ketiakellen@gmail.com \\ Daisy Schneider, UFRGS, daisy.schneider@gmail.com \\ Patricia Alejandra Behar, UFRGS, pbehar@terra.com.br \\ Arthur Adolfo, UFRGS, arthur_adolfo@hotmail.com
}

\begin{abstract}
Resumo. Este artigo trata da construção de um módulo para avaliação de competências e sua incorporação em um sistema de recomendação de objetos de aprendizagem baseado em competências. A integração do instrumento nesse sistema visa: 1) utilizar a avaliação em contextos educacionais na modalidade a distância como potencializador da prática docente apoiada em tecnologias digitais; e 2) colaborar na indicação de objetos de aprendizagem (OAs) baseados no perfil do aluno. Em ambas as questões, a finalidade é contribuir nos processos de aprendizagem dos alunos, especialmente na construção de competências.
\end{abstract}

Palavras-Chave: avaliação, competências, sistemas de recomendação.

\begin{abstract}
This article deals with the construction of a module for the Assessment of competences and its incorporation into a recommendation system of learning objects based on competences. The integration of the instrument into this system aims to: 1) use assessment in educational contexts in the distance modality as a potentializer of the teaching practice supported in digital technologies; and 2) collaborate in the indication of learning objects (LOs) based on the student profile. In both questions, the purpose is to contribute to the learning processes of the students, especially in the construction of competences.
\end{abstract}

Keywords: assessment, competences, recommendation system.

\section{Introdução}

O presente artigo tem como objetivo apresentar a construção do módulo de avaliação de competências em um sistema de recomendação de objetos de aprendizagem por competências, intitulado RecOAComp. Inicialmente, trata acerca do tema de avaliação de competências apontado por Sacristán (2011) como uma difícil tarefa, pois é uma prova de fogo que a proposta pedagógica terá que superar. Para ele, a avaliação das competências deve ser formativa e permanente. Neste caso, é preciso saber o que se avalia, isto é, quais competências específicas, e como o procedimento será feito, por exemplo, através de instrumentos em forma de situações-problemas.

Zabala e Arnau (2010) assinalam que diferentemente de uma avaliação focada apenas no conhecimento, na qual o docente verifica informações dos alunos sobre determinados conteúdos as competências assumem a complexidade de uma aprendizagem para a vida toda. $\mathrm{O}$ aluno, portanto, precisa responder a situações que simulem contextos para construir elementos ou recursos os quais lhes deem subsídios para aplicações posteriores diante de problemas reais.

Após a discussão quanto aos aspectos que sustentam este trabalho em termos de avaliação da aprendizagem, aborda-se o processo de construção do módulo para avaliação de competências e a sua incorporação ao sistema de recomendação que trata 
este artigo. Encerra-se com as considerações onde aponta-se, inclusive, as indicações sobre as próximas etapas de desenvolvimento do estudo.

\section{Avaliação de Competências e Sistemas de Avaliação}

Esta seção tem como objetivo apresentar os referenciais teóricos desta pesquisa. Inicialmente trata-se do conceito de avaliação de competências e, em seguida, descrevem-se alguns sistemas de avaliação de competências voltados para a educação.

\subsection{Avaliação de competências: conceitos}

Perrenoud (2000, p. 6) afirma que "é mais fácil avaliar conhecimentos de um aluno do que suas competências porque, para apreendê-las, deve-se observá-lo lidando com tarefas complexas, o que exige tempo e abre caminho à contestação". Para o autor deve-se criar estratégias de avaliações que possibilitem ao aluno desenvolvê-las. Já Sánchez e Ruiz (2011) destacam a importância de optar por uma avaliação que ocorra através da mobilização de conhecimentos, habilidades e atitudes de forma integral ao invés de realizá-la separadamente com cada elemento, visto que tal procedimento se mostra mais eficiente e eficaz. Assim, é preciso partir de situações-problemas, simulando contextos reais e dispondo de meios de avaliações específicos (Zabala; Arnau, 2010).

Clarke-Midura et al (2011, p. 235) consideram que: “desenvolver uma avaliação efetiva do desempenho com tarefas de investigação científica requer a análise dos processos cognitivos e das estruturas que contribuem com o desempenho das atividades". Por isso, o processo avaliativo deve utilizar mecanismos que permitam reconhecer se os esquemas de atuação aprendidos pelo sujeito podem ser úteis para superar situações reais em contextos reais. Portanto, avaliar competências é reconhecer a capacidade que um sujeito constrói para responder a cenários os quais apresentam probabilidade de acontecerem em sua vida real. Segundo Zabala e Arnau (2010), se o objetivo da avaliação das competências for somente verificar a forma como se resolve a situação, não auxiliará os sujeitos a melhorarem a competência. É preciso investigar o nível, através da realização de uma série de ações anteriores que permitam conhecer o grau de domínio do sujeito. O objetivo, portanto, é identificar as dificuldades dos alunos em busca de estratégias de ensino.

Conforme Tardif (1996 apud Perrenoud, 1999), a avaliação de competências, além da mobilização, deve considerar os conhecimentos prévios. Complementarmente, Zabala e Arnau (2010) concebem a avaliação a partir de indicadores de obtenção relativos à competência específica. Então, o que se pretende avaliar é o nível de aprendizagem. Cada indicador se refere a um ou mais elementos das competências, como diferentes conhecimentos, habilidades ou atitudes. A partir de atividades, simulando situações reais, é possível verificar os indicadores apropriados a cada um dos componentes da competência. Abaixo, a Figura 1, apresenta a inter-relação entre os diferentes elementos que configuram o processo de identificação dos indicadores a partir de uma situação problema e das correspondentes competências envolvidas. 


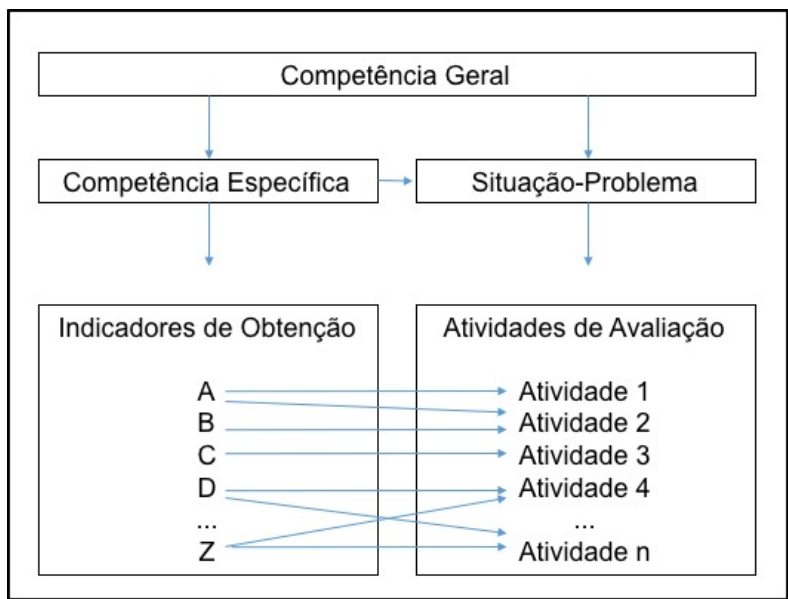

Figura 1. Esquema do processo de elaboração de atividades de avaliação Fonte: Zabala e Arnau (2010)

Zabala e Arnau (2010), ainda explicam que existe uma competência geral da qual se derivam as específicas. Para cada uma delas, devem ser definidos um ou vários indicadores de obtenção que permitem evidenciar o grau e o modo com o qual os alunos realizam a aprendizagem dos diferentes componentes, ou seja, dos conteúdos. Com o intuito de avaliar as competências, deve-se criar uma situação de aprendizagem que leve o aluno a compreender ambos os tipos de competências necessárias.

Portanto, Perrenoud (2001) adverte que não se constroem competências sem avaliá-las, mas esse procedimento não pode tomar a forma de provas, uma vez que um teste escrito é muito limitado, principalmente quando se busca avaliar as atitudes deste sujeito. Mon (2015) apresenta uma classificação de instrumentos para avaliação de competências, a saber: a) Testes Objetivos; b) Provas com perguntas curtas; c) Provas Cientifico-Matemáticas; d) Provas Orais; e) Simulação; f) Provas práticas para habilidades; g) Avaliação em laboratório; e h) Dossiê de aprendizagem, com todos os trabalhos dos alunos. Embora o autor apresente como opções provas e testes, vale destacar a contribuição dos demais instrumentos como bons meios de avaliação das competências por possibilitar a associação com cenários possíveis em situações do cotidiano do estudante.

Desta forma, com os recursos tecnológicos existentes, é possível transformar, através de uma série de ferramentas, as avaliações por competências em situações com maior realidade, que incluam tanto os resultados, quanto o registro das atividades. De acordo com Kuo et al (2013), durante décadas se identificou as vantagens das avaliações por meio das Tecnologias da Informação e Comunicação (TIC). Neste sentido, ao incorporar as TIC em uma abordagem de avaliação de competências, se procura criar modelos computacionais através de ferramentas e softwares que reproduzam situações mais reais. Assim, a partir deste resultado, o docente poderá planejar sua estratégia metodológica de construção das competências inseridas em processos de ensino e de aprendizagem sem a preocupação de classificar os sujeitos. A seguir, serão detalhados sistemas que realizam a avaliação de competências.

\subsection{Sistemas de avaliação de competências}

Nesta seção serão detalhados quatro sistemas que avaliam as competências na educação. Este levantamento teórico teve como objetivo auxiliar no desenvolvimento, na implementação e na integração do módulo ao sistema em questão. 
a) Modelo para Avaliação de Competências baseado em Projeto (Roque, 2004)

Desenvolvida em 2004, teve como objetivo auxiliar o professor e o aluno na avaliação de competências a partir do acompanhamento do desenvolvimento de um projeto voltado para a EAD. A ferramenta possui dois módulos, um para o professor e outro para o aluno, com funcionalidades diferentes. $O$ perfil do professor realiza o mapeamento das competências a serem desenvolvidas, definindo critérios, tipos de avaliação (diagnóstica e autoavaliação) e gerenciando as atividades realizadas. Para o mapeamento, o professor pode criar uma nova competência ou escolher uma que já esteja cadastrada no sistema. Já no perfil do aluno é possível visualizar as competências a serem desenvolvidas, as avaliações estabelecidas pelo docente e a atividade de projeto a ser realizada, recebendo feedback do professor se a competência foi ou não alcançada. Ao fim do curso, o aluno não recebe uma nota, mas um parecer contendo as competências desenvolvidas. Ambos os módulos possuem as mesmas funcionalidades com funções diferentes que são: Competências e Habilidades, Projeto, Avaliação Diagnóstica, Conceitos e Ajuda. A ferramenta foi um protótipo desenvolvido, cujos autores apontaram uma série de mudanças que deveriam ser realizadas, no entanto, a pesquisa somente foi realizada até esta etapa.

\section{b) Modelo A2COMP (Primo, 2004)}

Este modelo teve como objetivo descrever uma metodologia de cursos a distância e de avaliação de competências. A metodologia buscou promover $\mathrm{o}$ desenvolvimento de competências a partir de uma avaliação diagnóstica e formativa ao longo do curso, de acordo com quatro fases: 1) Identificação, 2) Avaliação Diagnóstica, 3) Acompanhamento realizado através do registro das atividades e 4) Final de Módulo através da rede de avaliação. A integração ocorreu no AVA TeleEduc por meio de um curso realizado para validação do A2COMP. A ferramenta utilizou como apoio a tomada de decisão na avaliação diagnóstica e na avaliação do módulo, o software Netica desenvolvido pela Norsys Software Corp. para gerar as redes bayesianas. No acompanhamento do curso foram utilizados aplicativos de edição de texto, planilha eletrônica e gerenciamento de banco de dados.

\section{c) AVACOMP - Avaliação de Competências (Brandão, 2012)}

A ferramenta teve como objetivo auxiliar o docente na avaliação das competências num ambiente de ensino e de aprendizagem. A ferramenta possui os perfis de aluno e professor, sendo esse último responsável por mapear as competências a serem desenvolvidas, definir os critérios de avaliação e acompanhar as atividades realizadas. $\mathrm{Na}$ tela inicial, o docente dispõe de quatro abas, quais sejam, Avaliação, Critérios, Relatório e Ajuda. O protótipo da ferramenta foi instalado em um servidor do Centro de Formação Profissional do SENAI-CE, liberando acesso para três docentes realizarem a validação com suas turmas. Após essa fase, o pesquisador constatou a necessidade de implementação de outras funcionalidades como o perfil de coordenador do curso (administrador), banco de competências com base nos currículos nacionais, criação de relatórios. Além disso, identificou aspectos de melhoraria de interface com o usuário; de integração do sistema de avaliação ao sistema da escola, a fim de buscar as turmas automaticamente; de criação da opção de novas competências pelo docente; de permissão da edição dos níveis de proficiência das competências; e de disponibilização dos resultados aos alunos. 


\section{d) DigiMima (Poldoja et al, 2014)}

DigiMima é um recurso de autoavaliação das competências digitais de docentes. Foi desenvolvido em 2014 para formação de professores na Estônia. Tem código aberto, sob licença Apache 2.0, de modo que todo o material está disponível podendo ser acessado livremente. Utiliza como indicadores-base o Modelo NETS para professores e está composta por vinte competências digitais divididas em cinco grupos. Cada competência é avaliada em uma escala de cinco pontos por meio de tarefas com a intenção de mapear os indicadores destas competências. As atividades de avaliação são divididas em três tipos: Autoavaliação; Avaliação de pares e Autorreflexão. Assim que o usuário finaliza todas as atividades, o sistema disponibiliza seu perfil de competências, através de um diagrama com o nível de cada uma das 20 competências. Atualmente, o DigiMima possui as seguintes funcionalidades: Criação do perfil do usuário; Autoavaliação das competências digitais; Autoteste; Fallback, ao errar um teste o sujeito retorna a questão até acertar; Seleção de itens randômicos; Revisão de atividades por pares; e Perfil de competências do sujeito.

Após a apresentação das quatro ferramentas de avaliação de competências, parte-se para a construção do módulo de avaliação das competências no RecOAComp.

\section{Construção do Módulo de Avaliação de Competências}

De acordo com os estudos realizados, compreende-se que é possível avaliar as competências por meio de situações a serem incorporadas em um sistema de recomendação com este foco. Neste capítulo, descreve-se brevemente o RecOAComp seguido da explanação sobre o módulo de avaliação construído.

\subsection{Recomendador de Objetos de Aprendizagem baseado em Competências}

O Recomendador de Objetos de Aprendizagem baseado em Competências, sobre o qual trata este artigo, permite realizar a filtragem desses materiais em conformidade com as competências que o usuário necessita construir ou reconstruir a partir de seu perfil. A Figura 2 demonstra a tela onde é efetuado o login no sistema.

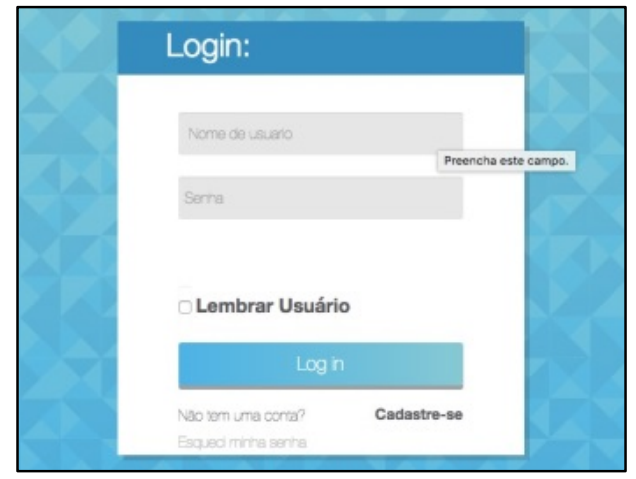

Figura 2. Tela inicial do RecOAComp Fonte: os autores

As pesquisas e o desenvolvimento do sistema de recomendação RecOAComp já perfazem seis anos. O objetivo do grupo de pesquisa é dispor à comunidade acadêmica uma ferramenta que possa auxiliar professores e alunos no processo de construção de competências. Atualmente o sistema conta com quatro tipos de filtragem: 
- Baseada em conteúdo: Realiza a filtragem aplicando o reconhecimento de elementos que podem atender a interesses comuns, implícitos ou explícitos. Foi o primeiro tipo de filtragem incluído no recomendador, sendo utilizadas as competências como variáveis. Desse modo, os OAs são recomendados ao sujeito a partir das competências que este necessite desenvolver ou aprimorar.

- Colaborativa: Realiza o processo de recomendação por meio do auxílio humano, decorrente da colaboração de grupos, que se interessam pelo mesmo elemento. Foi desenvolvida, neste caso, de modo a propiciar que o aluno possa avaliar uma escala de relevância de cada material a ele recomendado, conforme Figura 3. O sistema, por sua vez, adiciona essa informação (grau de pertinência de cada OA) ao processo das próximas recomendações.

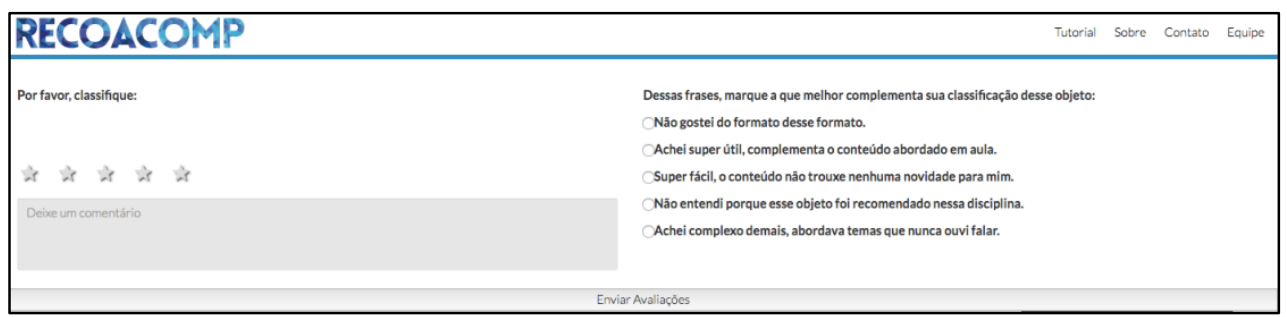

Figura 3 - Avaliação colaborativa do RecOAComp Fonte: os autores

- Híbrida: Relaciona mais de uma técnica de filtragem simultaneamente superando as limitações de cada modalidade e permitindo um resultado mais preciso. Desta forma, o sistema une as filtragens baseadas em conteúdo e colaborativa com variações de hibridismo, a fim de auxiliar no refinamento das indicações de materiais conforme o perfil do aluno.

- Contexto: São filtradas as preferências e contextos de cada aluno, como tempo disponível para estudos, local, estilo de aprendizagem e entre outros, auxiliando a seleção e indicação de recursos educacionais relevantes ao perfil e às necessidades de cada aluno.

Além das filtragens citadas, está em fase de implementação a filtragem por conhecimento no RecOAComp. Sua inclusão tem por objetivo possibilitar a relação dos conceitos com as competências cadastradas no sistema.

A primeira ação do professor no sistema é cadastrar sua atividade de ensino ${ }^{1}$, conforme ilustra a Figura 4. Nesse procedimento, irá informar as competências que a atividade de ensino ajudará a construir e em qual grau (em uma escala que situa os graus entre 0 e 4) poderão ser construídas pelos alunos.

\footnotetext{
${ }^{1}$ Optou-se por utilizar a nomenclatura corrente na Universidade, sendo considerados como atividade de ensino disciplinas, estágios curriculares e docentes, e trabalhos de conclusão.
} 


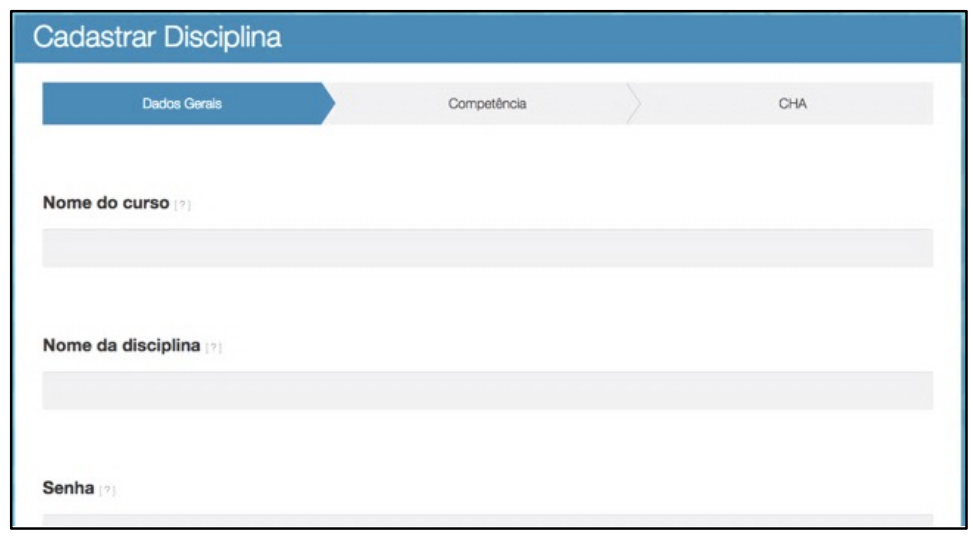

Figura 4. Cadastro de disciplina do RecOAComp

Fonte: os autores

Em seguida, o docente vincula Objetos de Aprendizagem (OAs) - já cadastrados no sistema ou cadastrando novos -, e quais competências estes podem ajudar a construir, a partir do seu conteúdo e atividades. Entende-se que um OA pode auxiliar na construção de diferentes competências e, da mesma forma, a competência registrada pode ter diferentes objetos de aprendizagem vinculados a ela.

Após tal procedimento, a atividade de ensino e os objetos ficam armazenados no banco de dados em um servidor da Universidade. Quando o estudante acessa o sistema e efetua seu cadastro, ele visualiza as competências listadas e deve informar o grau de construção (0 a 4) que entende possuir acerca delas. Assim, o aluno terá um perfil de competências relacionadas à atividade de ensino específica. Após estes procedimentos, o aluno pode solicitar uma recomendação, que consiste na indicação ao aluno de OAs a partir do cruzamento entre:

- Os graus de cada competência que a atividade de ensino pode possibilitar atingir, conforme apontado pelo professor;

- Os graus das competências informadas pelo aluno (autoavaliação); e

- Os graus de competências os quais podem ser alcançados pelos alunos a partir da exploração e do estudo dos objetos de aprendizagem, de acordo com a informação do professor que os cadastrou.

Assim, a filtragem é ativada e o aluno receberá materiais resultantes desse procedimento. Após a utilização dos OAs, o aluno pode fornecer um feedback ao sistema, avaliando de acordo com a escala Likert de 5 pontos, representado por um conjunto de estrelas, sua satisfação com a recomendação. Conforme o aluno vai interagindo com os objetos e aperfeiçoando as competências, ele pode retornar ao seu perfil e alterar o grau delas, modificando as próximas recomendações.

\subsection{Módulo de Avaliação de Competências}

A escolha por integrar um instrumento de avaliação de competências nesse sistema visa utilizar a avaliação em contextos educacionais na modalidade a distância como potencializador da prática docente apoiada em tecnologias digitais, como também colaborar na indicação de objetos de aprendizagem baseados no perfil do aluno, a partir das suas autoavaliações. Em ambas as questões, a finalidade é contribuir com os processos de aprendizagem dos alunos. Para alcançar o objetivo, o percurso de desenvolvimento inclui: 1) Modelagem, 2) Implementação, e 3) Validação. 
$\mathrm{Na}$ primeira etapa, houve a modelagem do módulo capaz de avaliar as competências digitais dos alunos da EAD, foram realizados estudos tendo por objetivo disponibilizar um panorama do processo de construção de competências dos alunos usuários do RecOAComp por meio de autoavaliações periódicas visualizadas através quadro e/ou gráfico. Além disso, foi realizado um levantamento teórico sobre avaliação de competências e sistemas existentes que realizam esta avaliação. Este levantamento teórico teve como intenção auxiliar na implementação do módulo.

A segunda etapa envolveu a implementação e desmembrou-se nas ações a seguir descritas: desenvolvimento da estrutura de construção das situações-problemas pelo docente, de acordo com a Figura 5; estabelecimento de periodicidade de disponibilização das situações aos alunos; e criação de um formato de visualização dos resultados da avaliação (comparativo) na visão de aluno e na visão de professor no sistema, por meio de gráfico.

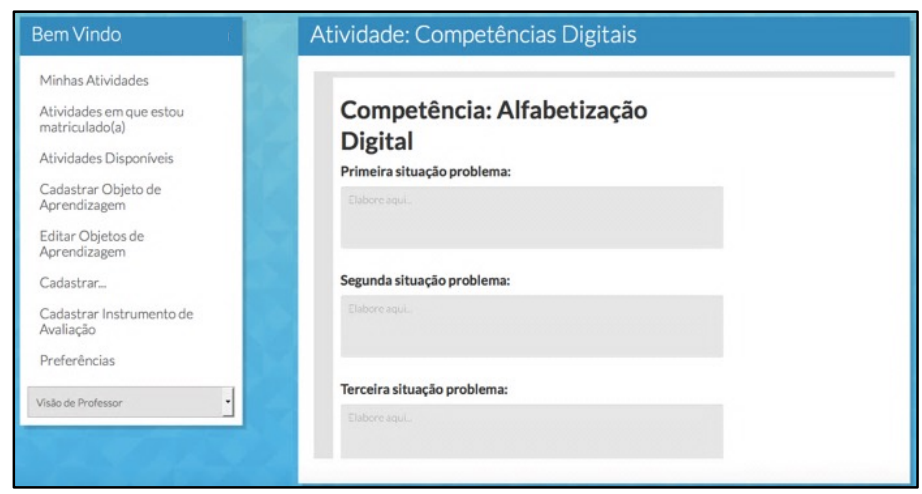

Figura 5. Exemplo da estrutura para construção das situações problemas do RecOAComp Fonte: os autores

A situação problema é avaliada de acordo com o CHA - Conhecimentos, Habilidades e Atitudes. A estrutura do questionário compõe situações-problema que contextualizam a competência ao mesmo tempo em que colocam uma questão de autoavaliação. Abaixo de cada situação-problema encontra-se uma lista de afirmações para os quais correspondem valores, de acordo com Figura 6. A partir disso, geram os graus de construção de competências. O aluno e o professor poderão ver o panorama (relatório) desse processo em forma de gráficos, os quais permitem visualizar os resultados de todas as avaliações. O docente pode programar até três momentos de avaliação (início, meio e fim) por atividade de ensino. Logo, houve estudos do ponto de vista pedagógico e de programação.

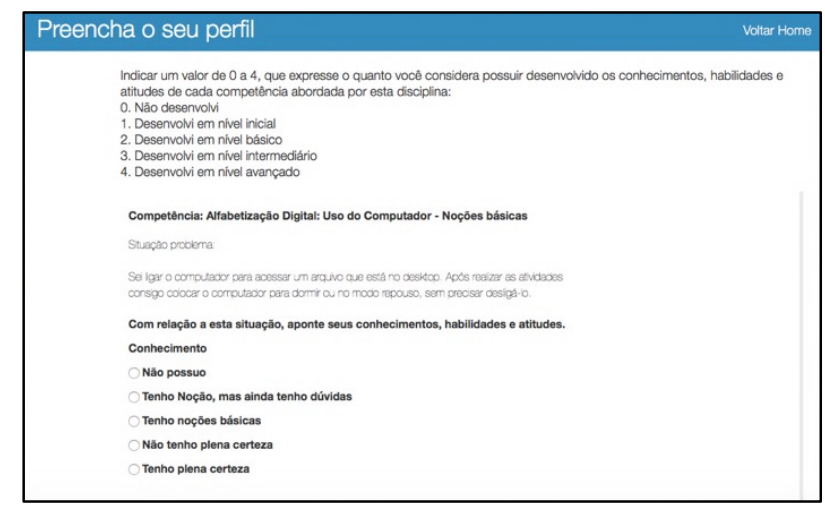

Figura 6 . Exemplo de questionário do RecOAComp Fonte: os autores 
A terceira etapa, validação está sendo realizada em 2017/1 em uma disciplina de pós-graduação.

\section{Considerações Finais}

Compreende-se que o módulo de avaliação desenvolvido pode ser um recurso importante em um sistema de recomendação de objetos de aprendizagem para a construção de competências. Serve como mecanismo de qualificação das recomendações, do acompanhamento do processo de aprendizagem dos alunos e da análise da própria prática pedagógica pelo professor. Nessa relação, indiretamente, revisam-se os objetos de aprendizagem disponíveis, assim como suas avaliações por filtragem colaborativa.

Com um módulo de avaliação, torna-se mais evidente para o aluno o andamento de seu processo de aprendizagem, tendo maior clareza dos pontos em que avançou e aqueles para os quais precisa dedicar mais atenção. Em Educação a Distância, esse dispositivo pode ser bastante importante na manutenção do aluno no curso/disciplina, bem como no processo de aprendizagem do professor atuante na modalidade ao avaliar seu fazer pedagógico com suporte das tecnologias, entre outras contribuições.

Pretende-se que esse sistema de recomendação de objetos de aprendizagem baseado em competências possa ser utilizado pela comunidade universitária da UFRGS, com o objetivo, em um futuro breve, de disponibilizá-lo junto aos ambientes virtuais de aprendizagem institucionais e/ou ao LUME. Associado a esses recursos, o RecOAComp pode qualificar o tipo de material disponibilizado a cada aluno de acordo com seu perfil (interesses e necessidades), de modo a aproximar a Universidade de umas das tendências da educação atual, isto é, a personalização do ensino. Contudo, o sistema está disponível à comunidade em geral para uso em suas disciplinas e cursos, com acesso pelo endereço http://www.recomendadorcomp.ufrgs.br/.

\section{Referências}

BRANDÃO, F. S. R. Ferramenta Computacional de Apoio ao processo de avaliação das competências na Educação professional. Fortaleza: MPCOMP/Instituto Federal de Educação, Ciência e Tecnologia do Ceará, 2012. 95f. Dissertação de Mestrado.

CLARKE-MIDURA, J. et al. Exploring the utility of a virtual performance assessment. In: SREE: Building and Education Science: Improving Mathematics and Science for all Students, 2011, Washington, EUA. Anais. Washington, EUA: Society for Research on Educational Effectiveness, 2011, p. 1-9.

KUO, E.; HULL, M.; GUPTA, A.; ELBY, A. How Students Blend Conceptual and Formal Mathematical Reasoning in Solving Physics Problems. Science Education, v. 97, n. 1, p. 32-57, 2013.

MON, F. M. E. La Competencia Digital Docente. Análisis De La Autopercepción Y Evaluacióndel Desempeño De Los Estudiantes universitarios De Educación Por Medio De Un Entorno 3d. Tortosa, Espanha: Universitat Rovira I Virgili/Departament de Pedagogia, 2015. 246f. Tesis Doctoral. 
PERRENOUD, P. Construir as competências desde a escola. Porto Alegre: Artmed, 1999. 96p.

PERRENOUD, P. Dez novas competências para ensinar. Porto Alegre: Artmed, 2000. 162p.

PERRENOUD, P. et al. (Org.). Formando professores profissionais: Quais estratégias? Quais competências? 2. ed. Porto Alegre: Artmed, 2001.232p.

POLDOJA, H. et al. Web-based self-and peer-assessment of teachers' digital competencies, World Wide Web, Victoria, Australia, v. 17, n. 2, p. 255-269, mar. 2014.

PRIMO, L. P. C. A. Metodologia para acompanhamento de cursos de EAD e avaliação de competências - A2COMP. Fortaleza: Universidade de Fortaleza, 2004. 145p. Dissertação de Mestrado.

ROQUE, G. O. B. Uma proposta de um modelo de avaliação de aprendizagem por competências para cursos à Distância baseados na web. Rio de Janeiro: Instituto de Matemática/UFRJ, 2004. 156f. Dissertação de Mestrado.

SACRISTÁN, J. G. et al. Educar por competências: o que há de novo? Porto Alegre: Artmed, 2011.264p.

SÁNCHEZ, A. V.; RUIZ, M. P. Evaluación de competencias genéricas: principios, oportunidades y limitaciones. Bordón. Revista de Pedagogía, Espanha, v. 63, n. 1, p. 147-170, 2011.

ZABALA, A.; ARNAU, L. Como aprender e ensinar competências. Porto Alegre: Artmed, 2010. 198p. 